\title{
12th FELASA SECAL Congress
}

\section{0-13 JUNE 2013}

International Convention Center

Barcelona, Spain

Attendance: $>1,900$ registrants from 53 countries

http://www.felasa2013.eu/

Twitter: @FELASA2013

The Federation of European Laboratory Animal Science Associations (FELASA) holds its Congress every 3 years, and this year's gathering - the largest laboratory animal science meeting in Europe-was organized by the Spanish Association of Laboratory Animal Science (SECAL). It was a happy coincidence that the Congress marked the 12th convention for both organizations.

The scientific program was structured into three different color-coded itineraries: blue for "Health, Welfare and Legal Issues;" green for "Animal Facility Managing;" and red for "LAS Applied Research." The motto "Better Science from Fewer Animals" was more than a beautiful phrase, as it really represented the core idea of many sessions and workshops. A number of discussions were focused on reduction strategies, such as the application of proper and accurate statistical designs, the use of mathematic models prior to the designing of animal studies, the sharing of data and the implementation of highresolution imaging techniques in animal facilities.

The last European Directive on the protection of animals used for scientific purposes, adopted in 2010 (Directive 2010/63/EU), was a hot topic because it has brought up a number of novelties, both conceptual and logistic, that the European countries must apply in a harmonized way. The scope of this Directive is wider than that of the previous one and includes new species, such as cephalopods. For this reason, a session of the FELASA SECAL Congress was devoted to this issue, in which the first guidelines for the care and welfare of cephalopods in research were launched. This work was the result of a coordinated effort co-chaired by FELASA, the Body Group, UK and CephRes-ONLUS.

There was also a session on severity assessment and endpoints, which was very well received by attendees, because speakers gave concrete advice for such assessment (which is mandatory under the new Directive) and for the establishment of humane endpoints in a variety of animal models. The conclusion talk of this session was given by a member of the Directorate-General for the Environment of the European Commission, who highlighted the importance of the attitude of professionals-considered greater than that of rules-to achieve high ethical standards in working with animals. The ethical review of projects under the new Directive was also analyzed and discussed in a separate session, and additional sessions focused on other issues related to that Directive, including

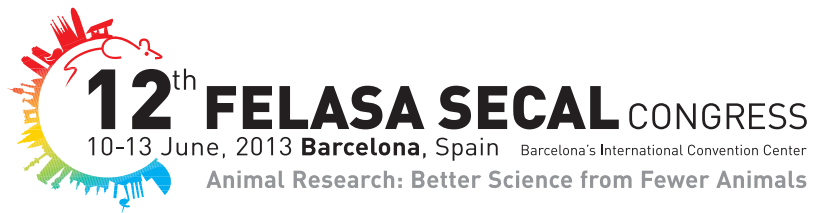

new approaches on education and training and implementation of this rule in Eastern European countries.

The three itineraries included a number of sessions focused on certain animal species whose use has been infrequent in the past but is currently increasing and might become more commonplace. Farm animals, poultry, wild animals and fish were highlighted in several sessions, which presented different perspectives including the management of aged animals, the design and management of facilities for such species, the refinement of disease models and the cryopreservation of sperm.

The institutional work of FELASA was showcased through a variety of activities: some FELASA documents were reviewed and discussed (for example, the FELASA guidelines for health monitoring of rodents and rabbits and the report of FELASA/ ESLAV/ECLAM Working Group on Severity); the current working groups were presented; and the AALAS-FELASA activities were updated. In general, the need to harmonize operating protocols across borders was a nearly unanimous opinion. The International Council for Laboratory Animal Science (ICLAS) was also wellrepresented in a session where current projects were presented and in the annual General Assembly. In addition, the official journal of FELASA, Laboratory Animals, organized an interesting workshop in which authors could meet the editor to analyze and discuss the focus and working procedures of the journal.

In general, the workshops had large audiences; in most cases, the demand was greater then the number of seats available. In addition to the indubitable interest of professionals in the interactive approaches to certain topics, the novelty of many of those topics was one of the most attractive factors. In one example, members of British social movements and journalists discussed with the audience how to present work with animals to society. And in a second example, three American research foundations presented funding opportunities unknown to many European professionals.

Three excellent plenary lectures gathered most attendees in the same room. First, María A. Blasco (Spanish National Cancer Research Center (CNIO), Spain) opened the Congress by giving an overview of her work on telomeres as therapeutic targets for cancer and aging. Cory Brayton (Johns Hopkins University, Baltimore, MD, US) talked about new advances on phenotyping procedures. And, finally, under the title "The two Rs approach," Timo Nevalainen (University of Eastern Finland) gave a lecture during the ceremony in which he received the FELASA 2013 Award. 